\title{
EPÍFITOS VASCULARES NA BACIA DO ALTO IGUAÇU, PARANÁ, BRASIL - COMPOSIÇÃO FLORÍSTICA
}

\author{
Vascular Epiphytes Checklist In The Upper Iguaçu River \\ Basin, Parana, Brazil
}

\author{
Rodrigo de Andrade Kersten ${ }^{1}$ \\ Yoshiko Saito Kunyioshi
}

\section{Resumo}

A partir de coletas em campo (14 estações), dados de herbário e de publicações, foram registradas para a região 348 espécies de epífitas vasculares distribuídas em 34 famílias e 97 gêneros. Duzentas e oito espécies foram observadas em campo, 123 em herbários (UPCB, MBM) e 17 retiradas de publicações. Destacam-se Orchidaceae (147 espécies), Bromeliaceae (39 espécies), Polypodiaceae (28 espécies), Piperaceae (24 espécies) e Hymenophyllaceae (15 espécies). A grande maioria das espécies foi classificada como holoepífita obrigatória. O menor número de epífitas obrigatórias foi observado entre as eudicotiledôneas. As magnoliides apresentaram o maior número de espécies facultativas e acidentais (46\%). A região floristicamente mais semelhante à estudada foi a Planície Costeira do Rio Grande do Sul, indicando a similaridade climática entre estas regiões, maior do que com regiões tropicais. A riqueza observada, tanto em espécies, como em gêneros e famílias, deve-se possivelmente à diversidade ambiental, ao grande número de sítios de coleta e ao contato entre duas formações (Floresta Ombrófila Densa e Floresta Ombrófila Mista).

Palavras-chave: Herbário; Epífitas vasculares; Holoepífita; Diversidade ambiental; Floresta ombrófila.

\begin{abstract}
Based on field (14 sites), herbarium and publication data we listed 348 vascular epiphyte species from 97 genera and 34 families. Field observation resulted in 208 species, 123 were registered on herbaria (UPCB and MBM), and 17 on published paper. Orchidaceae (147 species), Bromeliaceae (39 species), Polypodiaceae (28 species), Piperaceae (24 species), and Hymenophyllaceae (15 species) were the most diverse families. Most of the species were classified as true epiphytes. While the magnoliids had the heist number of facultative or accidental epiphytes (46\%) the eudicots had the smallest true epiphyte percentage (45\%). The region with flora most similar to the studied one was the Rio Grande do Sul coastal plain, possibly indicating climatic resemblance. The registered species, genera and families richness is possibly due to the environmental variety observed on the region, to the large studied site number, and the ecotone of two rich forests, the Dense and the subtropical ombrofilous forest.
\end{abstract}

Keywords: Herbarium; Vascular epiphyte; True epiphytes; Environmental variety; Ombrofilous forest.

\footnotetext{
Prof. Dr. PUCPR. Herbário HUCP. Centro de Ciências Biológicas e da Saúde, Rua Imaculada Conceição, 1155 - Prado Velho Curitiba - PR - CEP: 80215-901. kersten@pop.com.br

2 Prof.a Dr.a Sênior, Pós-graduação em Engenharia Florestal - UFPR. Av. Lothário Meissner, 632 - Jardim Botânico - Câmpus III, CEP 80210-170 - Curitiba - PR.
} 


\section{Metodologia}

Um exemplar, ao menos, de cada espécie encontrada fértil foi coletado para registro em herbário, exceções foram feitas para espécies muito comuns. Quando necessário, espécimes vegetativos foram coletados para fins de comparação e identificação. Espécies ainda não conhecidas foram coletadas quando férteis ou identificadas como morfoespécies, até que fosse possível sua identificação. Em alguns casos, indivíduos foram cultivados até estarem férteis. Quando observadas em mais de uma localidade, espécies férteis foram coletadas uma única vez, a não ser quando a ocorrência ainda não havia sido registrada para a formação vegetacional, unidade geológica ou para o município.

Foi também realizado levantamento de todas as espécies epífitas tombadas nos herbários MBM, UPCB e EFC, ocorrentes nos municípios considerados nas análises, além de levantamento de listagens publicadas para a região $(1,2,3,4)$.

As coletas de material botânico foram realizadas utilizando-se tesoura de poda para o material ao alcance da mão, tesoura de poda alta para materiais mais distantes e, quando necessário, o método de escalada simples ou com material próprio para montanhismo. Foi também utilizada uma escada de alumínio medindo $7,5 \mathrm{~m}$ para coleta e ancoragem dos equipamentos de escalada.

O material coletado foi identificado com o auxílio de literatura especializada, comparação com material já depositado nos herbários UPCB e MBM, e consulta a especialistas. Todo o material foi herborizado segundo procedimentos usuais em trabalhos florísticos e tombado nos herbários UPCB, MBM e EFC. As Angiospermas foram organizadas segundo o sistema APG (5) e a delimitação dos táxons pteridofíticos supra-específicos seguiu Moran (6). Pleurothallis (Orchidaceae), não obstante existência de publicações sugerindo sua divisão foi tratado como gênero único, por não estarem as novas propostas taxonômicas consolidadas, sendo observadas divergências entre autores $(7,8)$. Os nomes das espécies foram conferidos em W3tropicos (9).

Nas comparações florísticas utilizou-se o índice de Jaccard desconsiderando espécies exóticas ou introduzidas.

A partir da observação em campo, classificaram-se as espécies em seis categorias ecológicas, de acordo com sua ocorrência no meio epifítico (10). Holoepífito obrigatório (HLO), Holoepífito preferencial (HLP), Holoepífito facultativo (HLF), Holoepífito acidental (HLA), Hemiepífito primário (HMP) e Hemiepífito secundário (HMS).

\section{Resultados}

Foram registradas 348 espécies de epífitas vasculares distribuídas em 34 famílias e 97 gêneros, sendo 15 famílias, 26 gêneros e 94 espécies de pteridófitas, e 19 famílias (uma magnoliides, quatro monocotiledôneas e 14 eudicotiledôneas), 71 gêneros (três magnoliides, 49 monocotiledôneas e 19 eudicotiledôneas) e 254 espécies (24 magnoliides, 197 monocotiledôneas e 33 eudicotiledôneas) de angiospermas. Desse total, 208 espécies foram observadas em campo, 125 em herbários (UPCB, MBM) e o restante (17 espécies) retiradas de publicações $(1,2,3,4)$. 
TABELA 1 - Espécies observadas no levantamento florístico de epífitas vasculares na Bacia do Alto Iguaçu, seguidas das categorias ecológicas (HM = hemiepífito $-\boldsymbol{S}=$ secundário, $P=$ primário; $H L=$ holoepífito $-O=$ obrigatório $P=$ preferencial, $F=$ facultativo, $A=$ acidental), * indica espécies exóticas e forma de registro da espécie (Col. = coletada, MBM registrada no herbário MBM, UPCB - registrada no herbário UPCB, Publ. - publicada)

(Continua)

FAMíliA (número de espécies)

Espécie

Cat.

Reg.

\section{ARACEAE}

Anthurium acutum N.E. Br.

HLF

MBM

Anthurium longifolium Hort. ex Engl.

HLO

Col.

Philodendron bipinnatifidum Schott ex Endl.

HMP

MBM

Philodendron corcovadense Kunth

MBM

Philodendron cordatum Kunth

HMS

MBM

Philodendron loefgrenii Engl.

HMP

Col.

Philodendron missionum (Hauman) Hauman

HMP

MBM

Philodendron ochrostemon Schott

HMS

Col.

Philodendron propinquum Schott

HMP

MBM

\section{ASPLENIACEAE}

Asplenium auriculatum Mett.

HMS

$\mathrm{MBM}$

Asplenium auritum Sw.

HLP

MBM

Asplenium claussenii Hieron.

HLF

HLA

Col.

Asplenium gastonis Fée

HLP

Asplenium harpeodes Kunze

HLP

Col.

Asplenium inaequilaterale Willd.

HLF

Asplenium incurvatum Fée

HLP

Asplenium kunzeanum Klotzsch ex Rosenst.

HLF

HLP

Asplenium pseudonitidum Raddi

HLF

Asplenium scandicinum Kaulf.

Asplenium serra Langsd. \& Fisch.

HLP

HLF

Col.

Col.

ASTERACEAE

Erechtites valerianifolia (Wolf) DC.

\section{BALSAMINACEAE}

Impatiens walleriana Hook. f.

\section{BEGONIACEAE}

Begonia echinosepala Regel

Begonia fruticosa A. DC.

HLA

Col.

Col.

MBM

Col.

Col.

MBM

Col.

\section{BLECHNACEAE}

Blechnum asplenioides Sw.

Blechnum binervatum (Poir.) C.V. Morton \& Lellinger

\section{BROMELIACEAE}

Aechmea caudata Lindm.

Aechmea coelestis (K. Koch) E. Morren

HLF

Col.

Aechmea cylindrata Lindm.

HLA

Col.

Aechmea distichantha Lem.

HLA

Col.

Aechmea gamosepala Wittm.

HLA

Publ.

HMS

Col.

HLO

Col.

HLO MBM

HLO MBM

HLP Col.

HLO Col. 
Aechmea gracilis Lindm.

HLO

UPCB

Aechmea organensis Wawra

MBM

Aechmea ornata Baker

HLO

Col.

Aechmea recurvata (Klotzsch.) L.B. Sm.

HLP

Col.

Billbergia amoena (Lodd.) Lindl.

HLO

UPCB

Billbergia distachia (Vell.) Mez

HLO

Col.

Billbergia nutans Wendl.

HLO

Col.

Nidularium amazonicum (Baker) Lindm. \& E. Morren

MBM

Nidularium campo-alegrensis Leme

HLO

HLF

MBM

Nidularium innocentii Lem.

HLF

Publ.

Nidularium procerum Lindm.

HLF

Col.

Pitcairnia flammea Lindl.

HLO

MBM

Quesnelia imbricata L.B. Sm.

HLA

Col.

Tillandsia crocata (E. Morren) Baker

HLA

Col.

Tillandsia geminiflora Brongn.

HLO

Col.

Tillandsia linearis Vell.

Tillandsia mallemontii Glaz. ex Mez

HLO

Col.

Tillandsia recurvata (L.) L.

HLO

HLO

Col.

Tillandsia streptocarpa Baker

HLO

Col.

Tillandsia stricta Sol. ex Sims

HLO

MBM

Tillandsia tenuifolia L.

HLO

Col.

Tillandsia usneoides L.

HLO

Col.

Vriesea altodaserrae L.B. Sm.

HLO

Col.

Vriesea carinata Wawra

HLO

MBM

Vriesea ensiformis (Vell.) Beer

HLO

MBM

Vriesea friburgensis Mez

HLO

Col.

Vriesea gigantea Mart. ex Schult. f.

HLO

Col.

Vriesea guttata Linden \& André

Vriesea incurvata Gaudich.

HLO

Col.

HLO

Col.

Vriesea paraibica Wawra

HLO

Col.

Vriesea philippocoburgii Wawra

Vriesea platynema Gaudich.

HLO

MBM

HLO

Col.

Vriesea reitzii Leme \& A. Costa

HLO

Col.

Wittrockia cyathiforme (Vell.) Leme

HLO

Col.

\section{CACTACEAE}

HLO

Col.

Hatiora gaertneri (Regel) Barthlott

Hatiora salicornioides (Haw.) Britton \& Rose

HLO

MBM

Lepismium cruciforme (Vell.) Miq.

HLO

Col.

Lepismium houlletianum (Lem.) Barthlott

HLO

Col.

Lepismium lumbricoides (Lem.) Barthlott

HLO

Col.

Lepismium warmingianum (K. Schum.) Barthlott

HLO

Col.

Rhipsalis campos-potoana Loefgr.

Rhipsalis cereuscula Haw.

HLO

Col.

HLO

Col.

HLO

Col.

Rhipsalis floccosa Salm-Dick. ex Pfeiff.

HLO

Col.

Rhipsalis neves-armondii K. Schum.

HLO

Col.

Rhipsalis puniceo-discus G. Lindb.

HLO

MBM

Rhipsalis teres (Vell.) Steud. = capiliformis

HLO

Col.

\section{COMMELINACEAE}

Commelina robusta Kunth

HLA

Col.

Tradescantia fluminensis Vell.

HLA

Col. 
DENNSTAEDTIACEAE

Lindsaea botrychioides St.Hil.

\section{DRYOPTERIDACEAE}

Ctenitis falciculata (Raddi) Ching

Rumohra adiantiformis (G. Forst) Ching

\section{GESNERIACEAE}

Nematanthus tessmannii (Hoehne) Chautems

Nematanthus wettsteinii (Fritsch) H.E. Moore

Sinningia douglasii (Lindl.) Chautems

\section{GRAMMITIDACEAE}

Cochlidium punctatum (Raddi) L.E. Bishop

Cochlidium serrulatum (Sw.) L.E. Bishop

Lellingeria apiculata (Kunze ex Klotzsch) A.R. Sm. \& R.C. Moran

Lellingeria brevistipes (Mett. ex Kuhn) A.R. Sm. \& R.C. Moran

Lellingeria depressa (C. Chr.) A.R. Sm. \& R.C. Moran

Lellingeria organensis (Gardner) A.R.Sm. \& R.C. Moran

Lellingeria schenckii (Hieron.) A.R. Sm. \& R.C. Moran

Melpomene pilosissima (M. Martens \& Galeotti) A.R. Sm. \& R.C. Moran

Terpsichore achilleifolia (Kaulf.) A.R. Sm.

Terpsichore cultrata (Willd.) A.R. Sm.

Terpsichore reclinata (Brack.) Labiak

Zygophlebia longipilosa (C. Chr.) L.E. Bishop

GRISELINIACEAE

Griselinia ruscifolia (Clos) Taub.

\section{HYMENOPHYLLACEAE}

Hymenophyllum asplenioides (Sw.) Sw.

Hymenophyllum caudiculatum Mart.

Hymenophyllum hirsutum (L.) Sw.

Hymenophyllum magellanicum Wild.

Hymenophyllum polyanthos (Sw.) Sw.

Hymenophyllum pulchellum Schltdl. \& Cham.

Hymenophyllum vestitum (C. Presl) Bosch

Trichomanes anadromum Rosent.

Trichomanes anomalum Maxon \& C.V. Morton.

Trichomanes capillaceum $\mathrm{L}$. = pleopeltifoliatum

Trichomanes cristatum Kaulf

Trichomanes hymenoides Hedw.

Trichomanes polypodioides $\mathrm{L}$.

Trichomanes pyxidiferum L.

Trichomanes radicans $\mathrm{Sw}$.

\section{LOMARIOPSIDACEAE}

Elaphoglossum burchellii (Baker) C.Chr

Elaphoglossum chrysolepis (Fée) Alston

Elaphoglossum lingua (C. Presl) Brack.

Elaphoglossum ornatum (Mett. ex Kuhn) H. Christ

Elaphoglossum paulistanum Rosenst.

Elaphoglossum sellowianum (Klotzsch ex Kuhn) T. Moore

\section{LYCOPODIACEAE}

Huperzia acerosa (Sw.) Holub

Huperzia biformes (Hook.) Holub

Huperzia comans (Herter ex Nessel) B. Øllg. \& P.G. Windisch
HLA

Col.

HLA

HLP

Publ.

Col.

HLO

UPCB

HLO

Col.

HLO

Col.

HLO

Col.

HLO

MBM

HLO

Col.

HLO

MBM

Col.

MBM

HLO

Col.

HLO Col.

HLO Col.

HLO MBM

HLO Col.

HLO Col.

HLF

MBM

HLO

Col.

HLP

HLP

Col.

HLO

Col.

HLO

HLO

MBM

HLO

Col.

HLO

Col.

HLP MBM

HLO Col.

HLO MBM

HLO Col.

HLO Col.

HLO Col.

HLA MBM

HLO

MBM

HLO Col.

HLO Col.

HLO Col.

HLO Col.

HLO Col.

HLO MBM

HLO MBM

HLO Col. 
MELASTOMATACEAE

HLO MBM

Leandra carassana (DC.) Cogn. Leandra laxa Cogn.

\section{MORACEAE}

Ficus luschnathiana (Miq.) Miq.

HLA

Col.

Ficus monckii Hassl.

\section{MYRSINACEAE}

Myrsine gardneriana A.DC. Myrsine umbellata Mart.

HLA

Col.

\section{OLEACEAE}

Ligustrum lucidum W. T. Aiton

HMP

Col.

ONAGRACEAE

Fuchsia regia (Vell.) Munz

HMP

Publ.

\section{OPHIOGLOSSACEAE}

Ophioglossum palmatum L.

HLA

Col.

HLA

Col.

\section{ORCHIDACEAE}

Amblostoma armeniacum (Lindl.) Brieger ex Pabst

HLA

Col.

Barbosella australis (Cogn.) Schltr.

Barbosella miersii (Lindl.) Schltr.

Barbosella porschii (Kraenzl.) Schltr.

Bifrenaria harrisoniae (Hook.) Rchb.f.

Bulbophyllum granulosum Barb. Rodr. (Grande)

Bulbophyllum napellii Lindl. (pequeno)

HMP

Col.

Campylocentrum aromaticum Barb. Rodr.

Campylocentrum burchellii Cogn.

Campylocentrum rhomboglossum Hoehne \& Schltr.

HLO

Col.

Campylocentrum ulaei Cogn.

Capanemia adelaidae Porto \& Brade

Capanemia angustilabia Schltr.

HLO

MBM

HLO

Col.

HLO

MBM

HLO

MBM

HLO

Col.

HLO Col.

HLO Col.

HLO Col.

HLO Col.

HLO MBM

HLO Col.

HLO MBM

Capanemia australis (Kraenzl.) Schltr.

$\begin{array}{ll}\text { HLO } & \text { MBM } \\ \text { HLO } & \text { Col. }\end{array}$

Capanemia hatschbachii Schltr.

Capanemia superflua (Rchb. f.) Garay

HLO

Col.

Capanemia theresiae Barb.Rodr.

HLO

Col.

Cryptophoranthus langeanus (Kraenzl.) Garay

HLO

Col.

HLO

Cyclopogon sp.

HLF

Cyclopogon trifasciatus Schltr.

HLF

Dendrobium nobile Lindl.

HLO

Dichaea cogniauxiana Schltr.

HLO MBM

Dichaea pendula (Aubl.) Cogn.

HLO MBM

Dryadella edwallii (Cogn.) Luer

HLO MBM

Dryadella liliputiana (Cogn.) Luer

HLO Col.

Dryadella zebrina (Porsch) Luer

HLO MBM

Epidendrum caldense Barb. Rodr.

Col.

Epidendrum paniculatum Ruiz \& Pavon

HLO

MBM

Epidendrum proligerum Barb. Rodr.

HLF

Col.

Epidendrum secundum Jacq. (sin. ellipticum)

HLO

Col.

Col. 
Gomesa glaziovii Cogn.

Gomesa planifolia Klotzsch ex Rchb. f.

Gomesa recurva Lodd.

Grobya galeata Lindl.

Isabelia pulchella (Kraenzl.) Senghas \& Teusch.

Lankesterella ceracifolia (Barb. Rodr.) Ames

Lankesterella epiphyta (Barb. Rodr.) Mansf.

Leptotes unicolor Barb. Rodr.

Lophiaris pumila (Lindl.) Braem

Maxillaria acicularis Herb. ex Lindl.

Maxillaria cogniauxiana Hoehne

Maxillaria echinophyta Barb. Rodr.

Maxillaria heterophylla Hoehne

Maxillaria juergensii Schltr.

Maxillaria notylioglossa Rchb. f.

Maxillaria paulistana Hoehne

Maxillaria picta Hook.

Maxillaria vitelliniflora Barb. Rodr

Octomeria cf. alpina Barb. Rodr.

Octomeria chamaeleptotes Rchb. f.

Octomeria crassifolia Lindl.

Octomeria decumbens Cogn.

Octomeria elobata Schltr. ex Pabst

Octomeria gracilis Barb.Rodr.

Octomeria iguapensis Schltr.

Octomeria palmyrabellae Barb. Rodr.

Octomeria riograndensis Schltr.

Oncidium blanchetii Rchb. f.

Oncidium concolor Hook.

Oncidium curtum Lindl.

Oncidium fimbriatum Hoffmanns.

Oncidium fuscans Rchb. f.

Oncidium gardneri Lindl.

Oncidium hookeri Rolfe

Oncidium loefgrenii Cogn.

Oncidium longicornu Mutel

Oncidium longipes Lindl.

Oncidium montanum Barb. Rodr.

Oncidium paranaense Kraenzl.

Oncidium pulvinatum Lindl.

Oncidium raniferum Lindl.

Oncidium riograndense Cogn.

Oncidium sphegiferum Lindl.

Oncidium trulliferum Lindl.

Oncidium unicorne Lindl.

Oncidium uniflorum Booth

Oncidium varicosum Lindl. \& Paxton

Ornithophora radicans (Rchb.f.) Garay \& Pabst

Phymatidium aquinoi Schltr.

Phymatidium delicatulum Lindl.

Phymatidium hysteranthum Barb. Rodr.
HLO

HLO

HLO

HLO

HLO

HLO

HLO

HLO

HLO

HLO

HLO

HLO

HLO

HLO

HLO

HLO

HLO

HLO

HLO

HLO

HLO

HLO

HLO

HLO

HLO

HLO

HLO

HLO

HLO

HLO

HLO

HLO

HLO

HLO

HLO

HLO

HLO

HLO

HLO

HLO

HLO

HLO

HLO

HLO

HLO

HLO

HLO

HLO

HLO

HLO

HLO
MBM

MBM

Col.

Col.

Col.

Publ.

MBM

Col.

Col.

MBM

MBM

MBM

MBM

Col.

MBM

MBM

Col.

Col.

Col.

MBM

MBM

MBM

Col.

Col.

Col.

Col.

MBM

MBM

MBM

MBM

Col.

MBM

MBM

Col.

Col.

Col.

Col.

MBM

Col.

Col.

MBM

MBM

MBM

MBM

Publ.

MBM

MBM

MBM

MBM

Col.

UPCB 
Pleurothallis adenochila Loef.

Col.

Pleurothallis aveniformis Hoehne

Pleurothallis bacillaris Pabst

HLO

Col.

Pleurothallis bicristata Cogn.

Col.

Pleurothallis bleyensis Pabst

HLO

MBM

Pleurothallis bradei Schltr.

HLO

MBM

Pleurothallis butantanensis Hoehne \& Schltr.

HLO

MBM

Pleurothallis capanemae Barb. Rodr.

HLO MBM

Pleurothallis carinifera (Barb. Rodr.) Cogn

HLO

MBM

Pleurothallis colorata Pabst

HLO

MBM

Pleurothallis corticicola Schltr.

$\begin{array}{ll}\text { HLO } & \text { MBM } \\ \text { HLO } & \text { Col. }\end{array}$

Pleurothallis crepiniana Cogn.

Pleurothallis dryadum Schltr)

HLO

Col.

Pleurothallis gert-hatschbachii Hoehne

HLO

Col.

Pleurothallis grobyi Bateman ex Lindl.

HLO

MBM

Pleurothallis hatschbachii Schltr.

Pleurothallis heterophylla (Barb. Rodr.) Cogn.

HLO

Col.

HLO

Col.

Pleurothallis hygrophila Barb. Rodr.

HLO

MBM

Pleurothallis hypnicola Lindl.

HLO

Col.

Pleurothallis ipyrangana Schltr.

HLO

MBM

Pleurothallis klotzschiana Rchb. f.

HLO

MBM

Pleurothallis linearifolia Cogn.

HLO

MBM

Pleurothallis punctata Ker.Gaw.

HLO

MBM

Pleurothallis luteola Lindl.

HLO

Col.

Pleurothallis marginalis Rchb. $\mathrm{f}$.

HLO

Col.

Pleurothallis mattinensis Hoehne

HLO

Col.

Pleurothallis mentigera Kraenzl.

HLO

Col.

Pleurothallis mirabilis Schltr.

HLO

UPCB

Pleurothallis mouraei Cogn.

HLO

Col.

Pleurothallis mouraeoides Hoehne

HLO

MBM

Pleurothallis paranaensis Schltr.

HLO

MBM

Pleurothallis piraquarensis Hoehne

HLO

Col.

Pleurothallis piratiningana Hoehne

HLO

Col.

Pleurothallis platysemos Rchb. $\mathrm{f}$.

HLO

MBM

Pleurothallis pruinosa Lindl.

HLO

Col.

Pleurothallis recurva Lindl.

HLO

Col.

Pleurothallis sarracenia Luer

HLO

Col.

Pleurothallis saundersiana Rchb. f.

HLO

Col.

Pleurothallis saurocephala Lodd.

HLO

Col.

Pleurothallis seriata Lindl.

HLO

Col.

Pleurothallis sonderana Rchb. $\mathrm{f}$.

HLO

MBM

Pleurothallis sordida Kraenzl.

HLO

Col.

Promenaea paranaensis Schltr.

HLO

MBM

Promenaea xanthina Lindl.

HLO MBM

Prosthechea bulbosa (Vell.) W.E.Higgins

HLO Col.

Prosthechea fausta (Rchb. f.) W.E.Higgins

HLO Col.

Prosthechea fragrans (Sw.) W.E.Higgins

HLO

Col.

Psilochilus modestus Barb. Rodr.

HLO MBM

HLO MBM

Rodrigueziella gomezoides (Barb. Rodr.) Berman

HLO MBM

Rodrigueziopsis eleutherosepala (Barb. Rodr.) Schltr.

HLO

MBM

Scaphyglottis modesta (Rchb. f.) Schltr.

HLO

Publ. 
Sophronitis coccinea Rchb. f.

Stelis drosophila Barb.Rodr.

Stelis fraterna Lindl.

Stelis intermedia Poepp. \& Endl.

Stelis papaquerensis Rchb. f.

Stelis ruprechtiana Rchb.f.

Stelis triangularis Barb. Rodr.

Zygopetalum crinitum Lodd.

Zygopetalum maxillare Lodd.

Zygostates alleniana Kraenzl.

Zygostates dasyrhiza (Kraenzl.) Schltr.

\section{PIPERACEAE}

Peperomia alata Ruiz \& Pav.

Peperomia blanda (Jacq.) Kunth

Peperomia catharinae Miq.

Peperomia caulibarbis Miq.

Peperomia clivicola Yunk.

Peperomia corcovadensis Gardner

Peperomia delicatula Henschen

Peperomia elongata Kunth

Peperomia glabella (Sw.) A. Dietr.

Peperomia hilariana Miq.

Peperomia hispidula (Sw.) A. Dietr.

Peperomia psilostachya C. DC.

Peperomia quadrifolia (L.) Kunth

Peperomia reflexa Kunth

Peperomia rhombea Ruiz \& Pav.

Peperomia rubricaulis (Nees) A. Dietr.

Peperomia submarginata Yunck.

Peperomia subretusa Yunck.

Peperomia tetraphylla (G. Forst.) Hook. \& Arn.

Peperomia trineura Miq.

Peperomia trineuroides Dahlst.

Peperomia urocarpa Fisch. \& C.A. Mey.

Piper hispidum Sw.

Pothomorphe umbellata (L.) Miq.

\section{POLYPODIACEAE}

Campyloneurum acrocarpon Fée

Campyloneurum austrobrasilianum (Alston) de la Sota

Campyloneurum lapathifolium (Poir.) Ching

Campyloneurum minus Fée

Campyloneurum nitidum C. Presl

Campyloneurum repens (Aubl.) C. Presl

Microgramma percussa (Cav.) de la Sota

Microgramma squamulosa (Kaulf.) de la Sota

Microgramma tecta (Kaulf.) Alston

Microgramma vacciniifolia (Langsd. \& Fisch.) Copel

Niphidium crassifolium (L.) Lellinger

Pecluma paradiseae (Langsd. \& Fisch.) M.G. Price

Pecluma pectinatiformis (Lindl.) M.G.Prince
HLO

HLO

HLO

HLO

HLO

HLO

HLO

HLO

HLO

HLO

HLO

MBM

MBM

MBM

MBM

Col.

Col.

MBM

Col.

Col.

Col.

Col.

HLF

HLO

HLO

HLP

HLF

HLF

HLO

HLO

HLF

HLF

HLF

HLO

HLO

HLO

HLO

HLO

HLF

HLA

HLO

HLO

HLO

HLF

HLA

HLA

HLP

HLO

HLP

HLP

HLP

HLO

HLO

HLO

HLO

HLO

HLO

HLO

HLO
Col.

Col.

Col.

Col.

Col.

Col.

Col.

Publ.

Col.

Publ.

Col.

Col.

Col.

MBM

MBM

Col.

Col.

MBM

Col.

Col.

MBM

Publ.

Col.

Col.

Col.

Col.

MBM

MBM

Col.

UPCB

MBM

Col.

MBM

Col.

Col.

MBM

Col. 
Pecluma pruinosa (de la Sota) M.G.Price

Pecluma recurvata (Kaulf.) M. G. Price

Pecluma sicca (Lindm.) M. G. Price

Pecluma singeri (de la Sota) M. G. Price

Pecluma truncorum (Lindm.) M.G. Price

Pleopeltis astrolepis (Liebm.) E. Fourn.

Pleopeltis macrocarpa (Bory ex Willd.) Kaulf.

Pleopeltis pleopeltifolia (Raddi) Alston

Polypodium catharinae Langsd. \& Fisch.

Polypodium fraxinifolium Jacq.

Polypodium hirsutissimum Raddi

Polypodium latipes Langsd. \& Fisch.

Polypodium meniscifolium Langsd. \& Fisch.

Polypodium pleopeltidis Fée

Polypodium typicum Fée

PTERIDACEAE

Doryopteris nobilis (T. Moore) C. Chr.

\section{SELAGINELLACEAE}

Selaginella muscosa Spring

\section{SOLANACEAE}

Dyssochroma longipes (Sendtner) Miers Solanum corymbiflorum (Sendtn.) Bohs

Solanum sanctaecatharinae Dunal

\section{THELYPTERIDACEAE}

Thelypteris araucariensis Ponce

Thelypteris decusata (L.) Proctor

Thelypteris hispidula (Decne.) C.F. Reed

\section{THYMELAEACEAE}

Daphnopsis racemosa Griseb.

\section{URTICACEAE}

Urera baccifera (L.) Gaudich. ex Wedd.

\section{VITTARIACEAE}

Vittaria lineata (L.) Sm.

Vittaria scabrida Klotzsch ex Fée

\section{WOODSIACEAE}

Deparia petersenii (Kunze) M. Kato
HLO

HLO

HLO

HLF

HLP

HLO

HLO

HLO

HLO

HLO

HLO

HLF

HLO

HLO

HLO

HLA

HLA

Publ.

Col.

Col.

Col.

MBM

MBM

Col.

Col.

Col.

MBM

Col.

MBM

Publ.

Col.

MBM

Col.

Col.

HMP Publ.

HLA Publ.

HLA Publ.

HLA Col.

HLA Col.

HLA Col.

HLA Col.

HLA Publ.

HLO Col.

HLO Col.

HLA Col.
Dentre as famílias com maior riqueza (Tabela 2) destacou-se Orchidaceae, com 158 espécies (45\%) e 37 gêneros (47\%). Outras três famílias apresentaram mais de 20 espécies, 12 apresentaram apenas uma espécie e 21 famílias apresentaram apenas um gênero.

A grande maioria das espécies foi classificada como holoepífito obrigatório (Tabela 3) principalmente entre pteridófitas e
(Conclusão)

.


TABELA 2 - Riqueza específica em famílias epifiticas vasculares observadas no levantamento florístico da Bacia do Alto Iguaçu, Paraná

\begin{tabular}{|c|c|c|c|c|c|c|c|c|}
\hline Família & Spp & $\%$ & Família & Spp & $\%$ & Família & Spp & $\%$ \\
\hline Orchidaceae & 147 & 42 & Solanaceae & 3 & $<1$ & Dennstaedtiaceae & 1 & $\overline{<1}$ \\
\hline Bromeliaceae & 39 & 11 & Thelypteridaceae & 3 & $<1$ & Griseliniaceae & 1 & $<1$ \\
\hline Polypodiaceae & 28 & 8 & Begoniaceae & 2 & $<1$ & Oleaceae & 1 & $<1$ \\
\hline Piperaceae & 24 & 7 & Blechnaceae & 2 & $<1$ & Onagraceae & 1 & $<1$ \\
\hline Hymenophyllaceae & 15 & 4 & Commelinaceae & 2 & $<1$ & Ophioglossaceae & 1 & $<1$ \\
\hline Aspleniaceae & 12 & 3 & Dryopteridaceae & 2 & $<1$ & Pteridaceae & 1 & $<1$ \\
\hline Cactaceae & 12 & 3 & Melastomataceae & 2 & $<1$ & Selaginellaceae & 1 & $<1$ \\
\hline Grammitidaceae & 12 & 3 & Moraceae & 2 & $<1$ & Thymelaeaceae & 1 & $<1$ \\
\hline Araceae & 9 & 3 & Myrsinaceae & 2 & $<1$ & Urticaceae & 1 & $<1$ \\
\hline Lycopodiaceae & 7 & 2 & Vittariaceae & 2 & $<1$ & Woodsiaceae & & \\
\hline Lomariopsidaceae & 6 & 2 & Asteraceae & 1 & $<1$ & & & \\
\hline Gesneriaceae & 3 & $<1$ & Balsaminaceae & 1 & $<1$ & & & \\
\hline
\end{tabular}

Dentre as 11 famílias com mais de cinco representantes, quatro apresentaram apenas espécies holoepífitas características (Tabela 4). Em Orchidaceae, apesar de terem sido observadas também espécies facultativas e acidentais, 97\% de suas espécies foram incluídas nesta categoria. No outro extremo, Araceae apresentou a menor proporção de espécies classificadas nesta categoria. Aspleniaceae foi a família que apresentou o maior número de espécies facultativas e preferenciais pelo ambiente epifítico.

A região floristicamente mais semelhante à estudada foi a Planície Costeira do Rio Grande do Sul (11), muito embora todos os valores do índice de Jaccard (Tabela 5) para a comparação florística das regiões tenham sido muito baixos.

\section{Discussão}

A riqueza do componente epifítico observada no Alto Iguaçu pode ser considerada elevada, tanto quando comparada com outros levantamentos de flora epifítica, como com outros componentes das florestas. Enquanto Isernhagen (12) lista, para todo o Paraná, 682 espécies arbóreas, em 162 publicações, este estudo apresenta mais da metade deste número, observado em trecho bastante restrito do Estado. Se consideradas em conjunto com esta, os levantamentos da Ilha do Mel (13), Guaraqueçaba (14) e Pico Marumbi (15), são listadas quase 560 espécies de epífitas vasculares, apenas para o leste do Estado, em quatro publicações. Isto sugere que o número de epífitas vasculares no Estado possa exceder o número de espécies arbóreas, mesmo considerada toda a devastação das florestas nativas e a ausência de levantamentos anteriores ao ano de 1985. 


\section{TABELA 3 - Classificação, segundo categorias ecológicas, das espécies epifiticas no total e dentro dos táxons, eudicotiledônea, magnoliides, monocotiledônea e pteridófitas, das espécies registradas no levantamento das epifiticas vasculares do Alto Iguaçu, Paraná}

\begin{tabular}{|c|c|c|c|c|c|c|c|c|c|c|c|}
\hline \multirow{2}{*}{\multicolumn{2}{|c|}{ Categoria }} & \multicolumn{2}{|c|}{ Total } & \multicolumn{2}{|c|}{ Eudicot. } & \multicolumn{2}{|c|}{ Magnoliides } & \multicolumn{2}{|c|}{ Monocot. } & \multicolumn{2}{|c|}{ Pteridófitas } \\
\hline & & $\mathrm{n}$ & $\%$ & $\mathrm{n}$ & $\%$ & n & $\%$ & $\mathbf{N}$ & $\%$ & $\mathbf{n}$ & $\%$ \\
\hline \multirow[t]{4}{*}{ Holoepífito } & acidental & 30 & 9 & 12 & 36 & 3 & 13 & 4 & 2 & 11 & 12 \\
\hline & facultativo & 25 & 7 & 2 & 6 & 8 & 33 & 8 & 4 & 7 & 7 \\
\hline & obrigatório & 263 & 76 & 15 & 45 & 12 & 50 & 176 & 89 & 60 & 64 \\
\hline & preferencial & 18 & 5 & 0 & 0 & 1 & 4 & 2 & 1 & 15 & 16 \\
\hline Hemiepífito & & 12 & 3 & 4 & 12 & 0 & 0 & 7 & 4 & 1 & 1 \\
\hline Total & & 349 & 100 & 33 & 100 & 24 & 100 & 197 & 100 & 94 & 100 \\
\hline
\end{tabular}

Quando comparada com levantamentos da flora epifítica, convém descartar estudos pontuais e considerar apenas trabalhos mais amplos, como os da Reserva Ecológica de Macaé de Cima - RJ (16) da Serra da Juréia - SP (17) e da Ilha do Mel - PR (10). A primeira publicação apresenta cerca de 300 espécies, na Serra da Juréia são listadas cerca de 160 espécies enquanto na Ilha do Mel é citada a ocorrência de 190 espécies de epífitas vasculares. Cabe ressaltar que todos os trabalhos incluíram levantamentos de campo, assim como de material tombado em herbários.

A elevada diversidade pode ser explicada pela variação ambiental observada na região. Em pouco menos de $100 \mathrm{~km}$, passa-se de área conecta a Floresta Ombrófila Densa (F.O.D.) e, portanto, influenciada por vegetação tropical, para áreas com média de temperatura inferior a $12^{\circ} \mathrm{C}$ e sujeitas a geadas no inverno. A geomorfologia é também fator importante. Enquanto no primeiro planalto o rio Iguaçu corre por ampla planície aluvial, sobre geologia muito plana, passa para o segundo planalto quase sem planícies, correndo em regime encaixado, por cerca de $20 \mathrm{~km}$, nas formações Campo do Tenente e Furnas, até abrir-se novamente, na formação Mafra, com várzeas de mais de $500 \mathrm{~m}$.
As três famílias mais ricas (Orchidaceae, Bromeliaceae e Polypodiaceae), aqui representadas por 223 espécies (63\%), estão também entre mais ricas mundialmente $(18,19,20,21)$, assim como no Brasil (10). Orchidaceae, mundialmente, representa $10 \%$ da flora terrestre (22) e $70 \%$ da epífita (18); inclui $44 \%$ das espécies neste levantamento, número muito semelhante ao observado por Kersten e Silva (13). Já Araceae que, de acordo com Benzing (18), é a segunda maior família epífita, aqui foi registrada com apenas nove espécies, oito das quais observadas apenas no ecótono Floresta Ombrófila Densa/Mista, enquanto Hymenophyllaceae, décima segunda família em número de espécies (18), é a quinta maior neste estudo.

O número de famílias foi também bastante elevado, sendo superior ao observado em qualquer outra localidade (Ilha do Mel $=27$, Juréia $=23$, Macaé-de-Cima $=24$, Planície Costeira do Rio Grande do Sul = 28). Das 12 famílias observadas, em apenas uma destas localidades, seis estão no Iguaçu (Griselinaceae, Myrsinaceae, Pteridaceae, Thymelaeaceae, Urticaceae e Woodsiaceae), todas holoepífitas acidentais; três no Rio Grande do Sul (Apocynaceae, Cyclanthaceae e Psilotaceae) e duas em Macaé (Bignoniaceae e Bombacaceae). A Serra 
TABELA 4 - Classificação, segundo categorias ecológicas, das espécies epifiticas nas famílias com mais de um representante, na bacia do Alto Iguaçu, valores expressos em porcentagens ( $H L=$ holoepífito $-O=$ obrigatório $P=$ preferencial, $F=$ facultativo, $A=$ acidental, $H M=$ hemiepífito)

\begin{tabular}{lccccc}
\hline FAMÍLIAS & HLO & HLP & HLF & HLA & HM \\
\hline Araceae & 11 & -- & 11 & -- & 78 \\
Aspleniaceae & -- & 50 & 42 & 8 & -- \\
Begoniaceae & -- & -- & -- & 100 & -- \\
Blechnaceae & -- & -- & -- & 50 & 50 \\
Bromeliaceae & 82 & 5 & 8 & 5 & -- \\
Cactaceae & 100 & -- & -- & -- & -- \\
Commelinaceae & -- & -- & -- & 100 & -- \\
Dryopteridaceae & -- & 50 & -- & 50 & -- \\
Gesneriaceae & 100 & -- & -- & -- & -- \\
Grammitidaceae & 100 & -- & -- & -- & -- \\
Hymenophyllaceae & 73 & 20 & -- & 7 & -- \\
Lomariopsidaceae & 100 & -- & -- & -- & -- \\
Lycopodiaceae & 100 & -- & -- & -- & -- \\
Melastomataceae & -- & -- & -- & 100 & -- \\
Moraceae & -- & -- & -- & -- & 100 \\
Myrsinaceae & -- & -- & -- & 100 & -- \\
Orchidaceae & 97 & -- & 3 & -- & -- \\
Piperaceae & 50 & 4 & 33 & 13 & -- \\
Polypodiaceae & 79 & 18 & 4 & -- & -- \\
Solanaceae & -- & -- & -- & 67 & 33 \\
Thelypteridaceae & -- & -- & -- & 100 & -- \\
Vittariaceae & 100 & -- & -- & -- & -- \\
\hline
\end{tabular}

da Juréia não apresentou nenhuma família exclusiva, possivelmente em função de não ter sido realizado levantamento específico para a flora epifítica, fator que influencia no rigor das observações.

A grande diversidade de gêneros, também é destaque na florística, embora, neste caso, em número igual ao observado para a Planície Costeira do Rio Grande do Sul. No entanto, o número de gêneros exclusivos do Iguaçu é maior que o do Rio Grande do Sul (18 gêneros). As demais localidades (Ilha do Mel, Juréia e Macaé), respectivamente com 74, 68 e 88 gêneros, sendo 8,6 e 14 exclusivos. Possivelmente esta diversidade seja devida a dois fatores, o primeiro, a já citada diversidade ambiental regional, e segundo, o elevado número de sítios considerados, 14 levantamentos de campo, 15 retirados de publicações, além das espécies tombadas em herbários.

O predomínio de holoepífitos é regra em trabalhos no Brasil. A proporção de hemiepífitos, no entanto, é bastante variável, dependendo da tipologia florestal estudada. Em Floresta Ombrófila Densa, Fontoura et al. (16) registraram 7\% e de hemiepífitas, Schutz-Gatti (14) registrou $11 \%$ e Kersten e Silva (10) registraram 8\%; diversos estudos em florestas subtropicais (23, 2, 3), Kersten e Silva (24) e Rogalski e Zannin (25) não registraram nenhum hemiepífito em suas amostragens, sugerindo que a existência de poucas hemiepífitas neste levantamento seja reflexo da formação vegetacional, não favorável a esta categoria ecológica. 
TABELA 5 - Comparação florística (Índice de Jaccard e dendrograma de similaridade) para as regiôes consideradas na análise [(Alto) Iguaçu: este estudo; Ilha (do Mel): 10; (Serra da) Juréia: 17; Macaé (de Cima): 16; Planície (Litorânea do) Rio Grande do Sul: 11]

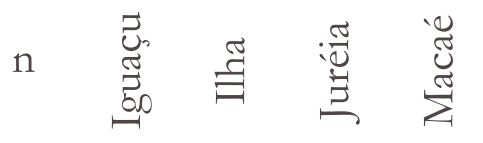

Ilha do Mel

189

0,14

Juréia

Macaé

160

$0,16 \quad 0,24$

308

0,17

258
Das 13 espécies de hemiepífitos listadas neste estudo, apenas seis foram registradas fora do ecótono Floresta Ombrófila Densa/Mista, destas, apenas três (Philodendron loefgrenii, Blechnum binervatum e Ficus luschnathiana) foram observadas em campo, o que corresponde a cerca de 1\% das espécies. Blechnum binervatum quase sempre inicia seu ciclo de vida no solo, parte das vezes perde a porção basal do sistema radicial/ caulinar passando a viver epidendricamente e a depender apenas de água e nutrientes provenientes do escorrimento pelos troncos das árvores suporte, por este motivo, ao contrário das outras três hemiepífitas, foi classificado como secundário. Em raros casos, sempre sobre Dicksonia sellowiana, foi também observado como hemiepífito primário. A figueira, embora tenha sido registrada com freqüência em diversas localidades, não foi observada "estrangulando" seu forófito em nenhum caso. Na grande maioria das vezes foram registrados indivíduos jovens de até um ou dois metros de altura.

A segunda categoria ecológica em número de espécies foi das holoepífitas facultativas. Muito embora não seja a regra $(1,4,24,13,25,14)$, resultado semelhante já foi observado (11). Mesmo espécies pouco comuns no ambiente epifítico (Erechtites valerianifolia, Piper hispidum, Myrsine gardneriana, M. umbellata, Solanum sanctaecatharinae) foram registradas crescendo, em muitos casos vigorosamente, sobre indivíduos arbóreos. M. gardneriana foi observada mais de uma vez, um dos indivíduos com mais de $5 \mathrm{~m}$ de altura. E. valerianifolia foi coletada a mais de 15 metros e com mais de 1,5 $\mathrm{m}$ de haste, P hispidum dava sinal de já ter passado por mais de uma fase reprodutiva. Esta categoria destacou-se principalmente entre as eudicotiledôneas, representado um quarto de suas espécies.

O conjunto das holoepífitas preferenciais é formado por espécies que ocorrem normalmente como epífitas, mas que podem também, sob condições especiais, ocorrer como terrícolas. Muitas vezes estão relacionadas a quedas de galhos, embora crescimentos espontâneos também tenham sido registrados, sobretudo, entre as pteridófitas, notadamente Aspleniaceae e Polypodiaceae. Algumas das espécies destas famílias já foram outrora classificadas como acidentais ou como obrigatórias $(24,13)$, porém as observações deste estudo levaram a uma classificação distinta.

O limite entre epífitas preferenciais, acidentais e facultativas, claro em teoria, pode ser confuso em campo, dificultando muitas vezes o enquadramento das espécies. Basear-se em literatura é prática pouco recomendada para esta situação, pois localmente espécies podem enquadrar-se em determinada classe e regionalmente encaixarem-se em outra. É o caso, entre outros, de Aechmea distichanta, Asplenium harpeodes e Rumohra adiantiformis, classificadas neste trabalho como preferenciais.

A. distichantha, embora nas florestas quase sempre ocorra como epífita, quando considerados outros ambientes, como afloramentos rochosos, poderia ser enquadrada facilmente como 
facultativa, mostrando até certa preferência pelo ambiente rupícola. As demais poderiam ser classificadas como facultativas a acidentais, na Serra do Mar, e como obrigatória, na região fitoecológica da Araucária, pois nestas florestas não foram registradas fora do dossel.

As epífitas facultativas pertencem mormente a famílias que possuem também espécies obrigatórias. Destacaram-se, nesta classe, Peperomia, no qual oito das 22 espécies enquadraram-se naquela categoria. Ao contrário, espécies acidentais, em geral, enquadram-se em famílias que não possuem epífitas, ou em famílias em que o epifitismo é incipiente. Quatorze das 19 famílias que abrigaram epífitas acidentais abrigaram apenas espécies desta categoria. Ambos os grupos, no entanto, foram mais bem representados entre as dicotiledôneas do que entre as pteridófitas ou monocotiledôneas.

A proporção das diferentes categorias de holoepífitos dentro dos táxons é indício do grau de especialização de cada grupo. Quando o nível de adaptação atinge certo patamar, a espécie pode passar a ter dificuldade em sobreviver fora de seu meio. Espécies fortemente adaptadas a retirar água da chuva, por exemplo, podem paralelamente perder a capacidade de resistir à umidade persistente e rapidamente apodrecer quando em contato com o solo (26). Isto não implica, contudo, que apenas espécies fortemente adaptadas sobrevivam nas copas.

As monocotiledôneas são, aparentemente, o grupo mais especializado ao hábito epifítico: além de mostrarem maior riqueza absoluta, possuem maior porcentagem de holoepífitos obrigatórios, sendo Orchidaceae (148 espécies) e Bromeliaceae (39 espécies) as grandes responsáveis pelos números nesta categoria. No entanto, análise mais detalhada das famílias mostra que, das quatro maiores famílias com 100\% de espécies obrigatórias, nenhuma está entre as monocotiledôneas, três são pteridófitas. Benzing (18) afirma que cerca de 75\% das orquídeas e $45 \%$ das bromélias ocorrem no ambiente epifítico, já em famílias como Grammitidaceae e Polypodiaceae este número pode ultrapassar $95 \%$.

As eudicotiledôneas parecem ser menos adaptadas para a vida desconecta ao solo, mesmo com $41 \%$ das famílias, o número de espécies foi baixo, ainda, a maioria das espécies foi classificada como não obrigatória, sendo também o grupo a apresentar a maior porcentagem de acidentais. Além de classificadas percentualmente menos como obrigatórias, possuem menos espécies em cada família. Entre as monocotiledôneas, quatro das três famílias possuem mais de cinco espécies, entre as pteridófitas são seis das 15 famílias e entre a eudicotiledôneas são apenas duas das 14 famílias, todas as 12 restantes com três ou menos espécies.

A baixa similaridade entre as localidades é devida possivelmente à diversidade gama de Orchidaceae. Das 800 espécies registradas nos cinco trabalhos (este estudo, 16, 10, 17, 14), 410 (52\%) são orquídeas, destas, 280 (67\%) ocorrem em apenas uma localidade. No total, 500 espécies (63\%) foram observadas em apenas um levantamento. Este grau de restrição tem sido um dos principais parâmetros utilizados para a previsão de números de espécies vegetais (27). Diversos autores já suscitaram discussões sobre o número de orquídeas, cuja menor estimativa previu 15.000 espécies (18) e a mais otimista 30.000 (21). Myers (27) chegou a estimar em 20.000 o número de plantas na floresta atlântica. O alto índice de espécies observadas em uma única localidade e a aparente "disjunção" apontada pelo índice de Jaccard sugere que o dossel, apesar do elevado número de espécies, ainda está longe de ser bem conhecido. Inexistem levantamentos da flora epifítica em número suficiente para uma descrição qualitativa adequada da flora que habita as copas das árvores dessas florestas.

A maior similaridade entre a região do Alto Iguaçu e a Planície Costeira do Rio Grande do Sul, assim como a menor similaridade com a Ilha do Mel, refletem, possivelmente, a similaridade climática. Ambas localizam-se sob clima subtropical, superúmido, sem período seco, com invernos rigorosos e com média anual entre 10 e $15^{\circ} \mathrm{C}$. A Ilha do Mel, por sua vez, está localizada em zona de clima tropical superúmido, não sujeita a geadas, também sem período seco e temperatura média anual de $21^{\circ} \mathrm{C}$.

A região do Alto Iguaçu apresenta-se muito rica em espécies, gêneros e famílias de epífitas. É o trabalho que inclui a maior listagem de espécies para o Brasil e para o mundo. A riqueza deve-se, entre outros fatores, à diversidade ambiental e ao contato entre duas formações (Floresta Ombrófila Densa e Floresta Ombrófila Mista). Orchidaceae é responsável por cerca de $45 \%$ das espécies e $40 \%$ dos gêneros. Pleurothallis 
(latu sensu), gênero mais rico, representou 15\% das espécies. A grande maioria (78\%) das espécies foi classificada como holoepífito obrigatório, a segunda categoria ecológica mais numerosa foram as holoepífitas acidentais. A região mais semelhante floristicamente à estudada foi a planície costeira do Rio Grande do Sul.

\section{Agradecimentos}

Aos funcionários e colaboradores do Museu Botânico Municipal, em especial ao Sr. Gerdt Hatschbachii, Osmar Ribas e Ronaldo Kummrow e aos colegas que foram a campo comigo, Carina Kosera, Daniel Gaioto, entre outros.

\section{Referências}

1. Borgo M, Silva SM. Epífitos vasculares em fragmentos de Floresta Ombrófila Mista, Curitiba, Paraná, Brasil. Revista Brasileira de Botânica. 2003; 26:391-401.

2. Cervi AC, Dombrowski LTD. Bromeliaceae de um capão de floresta primária do Centro Politécnico de Curitiba Paraná, Brasil. Fontqueria. 1985; 9:09-11.

3. Cervi AC, Acra LA, Rodrigues L, Train S, Ivanchechen SL, Moreira ALOR. Contribuição ao conhecimento das epífitas (exclusive Bromeliaceae) de uma floresta de araucária do primeiro planalto paranaense. Ínsula. 1988; 18:75-82.

4. Dittrich VAO; Kozera C; Silva SM. Levantamento florístico de epífitos vasculares no Parque Barigüi, Paraná, Brasil. Iheringia (Série Botânica). 1999; 52:11-22.

5. Stevens PF. Angiosperm Phylogeny Website. [serial on the Internet]. 2001 [cited 2005 Maio 20]. Disponível em: http://www.mobot.org/ MOBOT/research/Apweb.

6. Moran RC. Clave para las familias de pteridofitas. Flora Mesoamericana. Psilotaceae a Salvinaceae. Ciudad de Mexico: [s. n.]; 1995. v. 1.
7. Luer CA. Icones Pleurothallidinarum XXVI: Pleurothallis subgenus Acianthera and three allied subgenera; A Second Century of New Species of Stelis of Ecuador; Epibator, Ophidion, Zootrophion. Missouri Botanic Garden Monographs; 2004. p. 95.

8. Pridgeon AM, Chase MW. A phylogenetic reclassification of Pleurothallidinae (Orchidaceae). Lindleyana. 2001; 16:235-271.

9. W3tropicos - Missouri Botanical Garden's VAST (VAScular Tropicos) nomenclatural database. [serial on the Internet]. 2005 [cited 2005 Jan. 12] Disponível em http://mobot.mobot.org/ W3T/Search/vast.html.

10. Kersten RA. Epifitismo vascular na bacia do alto Iguaçu, Paraná [Tese]. Curitiba: Universidade Federal do Paraná; 2006.

11. Waechter JL. O epifitismo vascular na planície costeira do Rio Grande do Sul. São Carlos [Tese]. São Carlos: Universidade Federal de São Carlos; 1992.

12. Isernhagen, I. A fitossociologia florestal no Paraná e os programas de recuperação de áreas degradadas: uma avaliação [Dissertação]. Curitiba: Universidade Federal do Paraná; 2001.

13. Kersten, RA; Silva, SM. Forística e estrutura de comunidades de epífitas vasculares da planície litorânea. In: Marques M. C. M, Britez R. M, editor. História natural e conservação da Ilha do Mel. Curitiba: Universidade Federal do Paraná; 2005.

14. Schütz-Gatti, AL. O componente epifítico vascular na Reserva Salto Morato, Guaraqueçaba. PR. [Dissertação]. Curitiba: Universidade Federal do Paraná; 2000.

15. Petean MP. Florística e estrutura dos epífitos vasculares em uma área de Floresta Ombrófila Densa Altomontana no Parque Estadual do Pico do Marumbi, Morretes, Paraná, Brasil [Dissertação]. Curitiba: Universidade Federal do Paraná; 2003.

16. Fontoura T, Sylvestre LS, Vaz MAS, Vieira CM. Epífitas vasculares, hemiepífitas e hemiparasitas da Reserva Ecológica de Macaé de Cima. In: Lima, H.C, Guedes-Bruni, RR. (Eds.). Serra de Macaé de Cima: Diversidade florística e conservação da Mata Atlântica. Rio de Janeiro: Jardim Botânico do Rio de Janeiro; 1997. 
17. Mamede MCH, Cordeiro I, Rossi L. Flora vascular da Serra da Juréia, Município de Iguape, São Paulo, Brasil. Boletim do Instituto de Botânica. 2001; 15:63-124.

18. Benzing, DH. Vascular epiphytes: general biology and related biota. Cambridge: Cambridge University Press; 1990.

19. Gentry AH, Dodson CH, Diversity and biogeography of neotropical vascular epiphytes. Ann. Missouri. Bot. Gdns. 1987; 74:205-223.

20. Kress JW. The systematic distribution of vascular epiphytes: an update. Selbyana. 1986; 9:2-22.

21. Madison M. Vascular epiphytes: their systematic occurrence and salient features. Selbyana. 1977; $2: 1-13$.

22. Atwood JT. The size of Orchidaceae and the systematic distribution of epiphytic orchids. Selbyana. 1986; 9:171-186.

23. Aguiar LW, Citadini-Zanette V, Martau L, Backes A. Composição florística de epífitos vasculares numa área localizada nos municípios de Montenegro e Triunfo, Rio Grande do Sul, Brasil. Iheringia (Série Botânica). 1981; 28:55-93.
24. Kersten RA, Silva SM. Florística e estrutura do componente epifítico vascular em floresta ombrófila mista aluvial do rio Barigüi, Paraná, Brasil. Revista Brasileira de Botânica. 2002; 25(3):259-267.

25. Rogalski JM, Zanin EM. Composição florística de epífitos vasculares no estreito de Augusto César, Floresta Estacional Decidual do Rio Uruguai, RS, Brasil. Revista Brasileira de Botânica. 2003; 26:551-556.

26. Nadkarni NM. Longevity of fallen epiphytes in a Neotropical Montane forest. Ecology. 1993; 74:265-269.

27. Myers N, Mittermeier RA, Mittermeier CG, Fonseca GAB, Kent J. Biodiversity Hotspots for conservation priorities. Nature. 2000; 403:853858. 